\title{
Variabilité démographique de Saba senegalensis (A. DC.) Pichon suivant le gradient climatique au Burkina Faso
}

\author{
Blaise KABRÉ ${ }^{1}$ \\ Mamounata Belem/OUÉdRAOGO ${ }^{2}$ \\ Benjamin LANKOANDÉ ${ }^{3}$ \\ Amadé OUÉDRAOGO ${ }^{1}$ \\ ${ }^{1}$ Université Joseph Ki-Zerbo \\ Laboratoire de biologie \\ et écologie végétales \\ Département de biologie végétale \\ et physiologie végétale \\ Unité de formation et de recherche \\ en Sciences de la vie et de la Terre \\ (UFR/SVT) \\ 03 BP 7021, Ouagadougou 03 \\ Burkina Faso

${ }^{2}$ Centre national de recherche
scientifique et technologique
Institut national de l'environnement
et de recherches agricoles
Département Environnement et Forêt
03 BP 7047 , Ouagadougou 03
Burkina Faso
${ }^{3}$ Université de Fada N'Gourma
BP 54
Fada N'Gourma
Burkina Faso

\section{Auteur correspondant /} Corresponding author: Blaise KABRÉ

- kabreblaisetin@gmail.com

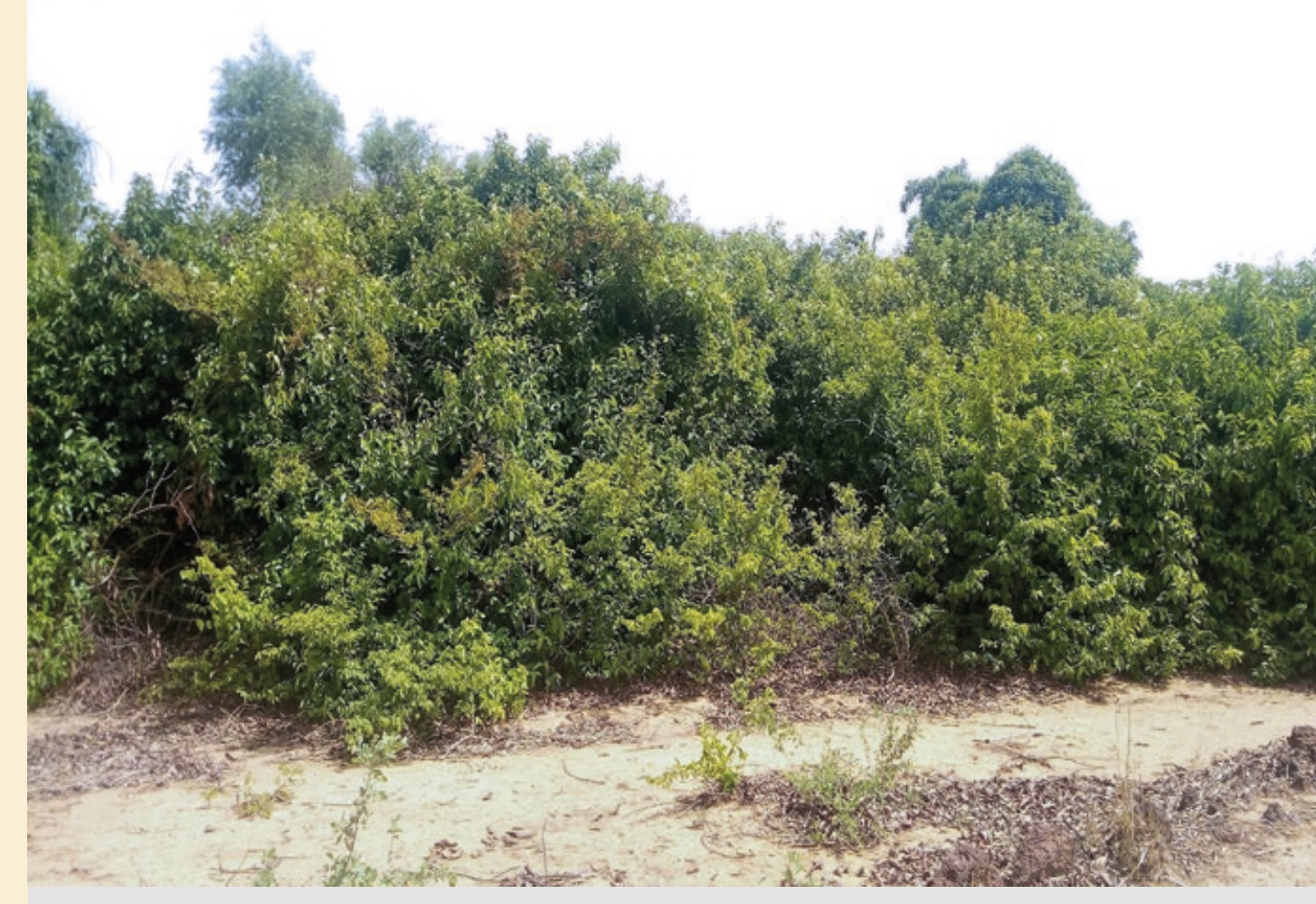

Photo 1.

Population de Saba senegalensis dans une savane de la zone sahélienne. Photo B. Kabré.

Doi : 10.19182/bft2020.345.a31930 - Droit d'auteur (c) 2020, Bois et Forêts des Tropiques - (c) Cirad - Date de soumission : 15 mars 2019 ; date d'acceptation : 13 mai 2020 ; date de publication : $1^{\text {er }}$ novembre 2020.

\section{cirad (a)}

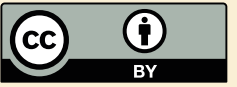

Citer l'article / To cite the article

Kabré B., Belem/Ouédraogo M., Lankoandé B., Ouédraogo A., 2020. Variabilité démographique de Saba senegalensis (A. DC.) Pichon suivant le gradient climatique au Burkina Faso. Bois et Forêts des Tropiques, 345 : 73-83. Doi : https://doi.org/10.19182/bft2020.345.a31930 


\section{RÉSUMÉ}

\section{Variabilité démographique de Saba senegalensis (A. DC.) Pichon suivant le gradient climatique au Burkina Faso}

Saba senegalensis (A. DC.) Pichon est une liane ligneuse de la famille des Apocynaceae. Elle est aussi appelée liane goïne. Les produits forestiers non ligneux obtenus à partir de cette espèce ont une grande importance socio-économique au Burkina Faso. Cependant, les caractéristiques démographiques de l'espèce sont peu connues bien que nécessaires pour mettre en place des programmes de gestion durable. La présente étude a pour objectif d'évaluer les caractéristiques démographiques de $S$. senegalensis suivant le gradient climatique au Burkina Faso. Un échantillonnage aléatoire orienté a conduit à installer des placeaux de $1000 \mathrm{~m}^{2}$ pour l'inventaire des populations. Les mesures dendrométriques ont concerné le diamètre de la tige au collet et à $1,30 \mathrm{~m}$ du sol pour les individus adultes et les diamètres à $20 \mathrm{~cm}$ du sol et au collet, respectivement pour les jeunes plants et les plantules. L'indice de Green, la densité, le diamètre moyen des tiges et la surface terrière ont été calculés et comparés entre les zones climatiques. Les résultats ont montré une distribution agrégative de $S$. senegalensis révélée par l'indice de Green. La densité, le diamètre moyen et la surface terrière ont varié significativement $(p<0,05)$ entre les zones climatiques. Les fortes densités des sujets adultes de $S$. senegalensis se trouvent dans les zone sahélienne et soudanienne. Le modèle linéaire généralisé montre que la température et les précipitations ont une influence sur l'établissement de la régénération de $S$. senegalensis. La structure démographique a révélé une abondance de juvéniles qui représentent un important potentiel de renouvellement des populations. S. senegalensis montre une certaine résilience de ses populations ; mais, du fait de la péjoration climatique et de la pression anthropique, la conservation de l'espèce dans son habitat naturel reste aléatoire.

Mots-clés : distribution spatiale, fruitier sauvage, liane goïne, structure des populations, zone semi-aride, Burkina Faso.

\section{ABSTRACT}

\section{Demographic variability of Saba senegalensis (A. DC.) Pichon according to the climate gradient in Burkina Faso}

Saba senegalensis (A. DC.) Pichon, also known as the weda vine, is a woody vine from the Apocynaceae family. The non-timber forest products obtained from this species are of major socio-economic importance in Burkina Faso, but its demographic characteristics are little known despite their importance for the implementation of sustainable management programmes. This study assessed the demographic characteristics of $S$. senegalensis according to the climate gradient in Burkina Faso. Targeted random sampling was used to establish $1,000 \mathrm{~m}^{2}$ plots for a population inventory. Dendrometric measurements of stem diameter were made at the root collar and at $1.30 \mathrm{~m}$ above the ground for adult individuals, $20 \mathrm{~cm}$ from the ground for young plants and at the root collar for seedlings. The Green index, density, average stem diameter and basal area were calculated and comparisons made between climatic zones. The results showed a clumped distribution of S. senegalensis according to the Green index. Density, average diameter and basal area varied significantly $(p<0.05)$ between climatic zones. High densities of adult $S$. senegalensis are found in the Sahelian and Sudanian zones. The general linear model shows that temperature and precipitation have an influence on the establishment and regeneration of S. senegalensis. The demographic structure shows an abundance of juvenile plants with high potential for the renewal of populations. Although S. senegalensis populations show a degree of resilience, the deteriorating climate and human pressures are jeopardising the conservation of the species in its natural habitat.

Keywords: spatial distribution, wild fruit tree, weda vine, population structure, semi-arid zone, Burkina Faso.

\section{B. LANKOANDÉ, A. OUÉDRAOGO}

\section{RESUMEN}

\author{
Variabilidad demográfica de Saba \\ senegalensis (A. DC.) Pichon según \\ el gradiente climático en Burkina Faso
}

Saba senegalensis (A. DC.) Pichon es una liana leñosa de la familia de las Apocynaceae. También se conoce como liana de Goin. Los productos forestales no leñosos obtenidos a partir de esta especie tienen una gran importancia socioeconómica en Burkina Faso. Sin embargo, las características demográficas de la especie son poco conocidas, aunque necesarias para implantar programas de gestión sostenible. El presente estudio tiene como objetivo evaluar las características demográficas de la $S$. senegalensis según el gradiente climático en Burkina Faso. Una muestra aleatoria orientada lleva a instalar parcelas de $1000 \mathrm{~m}^{2}$ para inventariar las poblaciones. Las medidas dendrométricas estudiaron el diámetro del tallo en el cuello de la raíz y a 1,30 $\mathrm{m}$ del suelo para los individuos adultos, y los diámetros a $20 \mathrm{~cm}$ del suelo y en el cuello de la raíz, respectivamente para las plantas jóvenes y las plántulas. Se calculó el índice de Green, la densidad, el diámetro medio de los tallos y el área basal, y se compararon entre las zonas climáticas. Los resultados mostraron una distribución agregativa de S. senegalensis revelada por el índice de Green. La densidad, el diámetro medio y el área basal variaron significativamente $(p<0,05)$ entre las zonas climáticas. Las fuertes densidades de sujetos adultos de $S$. senegalensis se encuentran en las zonas saheliana y sudanesa. El modelo lineal generalizado muestra que la temperatura y las precipitaciones influyen en la regeneración de la $S$. senegalensis. La estructura demográfica reveló la abundancia de ejemplares jóvenes, que representan un importante potencial de renovación de las poblaciones. S. senegalensis muestra una cierta resiliencia en sus poblaciones, pero a causa del empeoramiento climático y de la presión antrópica, la conservación de la especie en su hábitat natural resulta aleatoria.

Palabras clave: distribución espacial, frutal salvaje, liana de Goin, estructura de las poblaciones, zona semiárida, Burkina Faso. 


\section{Introduction}

En Afrique subsaharienne, de nombreuses espèces ligneuses fournissent des produits forestiers non ligneux (PFNL) qui représentent une contribution substantielle à la sécurité alimentaire et nutritionnelle et à l'amélioration des conditions de vie des populations locales (Lykke et Padonou, 2019). L'exploitation des PFNL génère des revenus qui concourent à l'amélioration des conditions socio-économiques des ménages ruraux et à la croissance des économies nationales (Assogbadjo et al., 2012 ; Vodouhê et al., 2009).

Durant les dernières décennies, les zones semi-arides ont été marquées par une dégradation accélérée des conditions environnementales, rendant fragile l'équilibre des écosystèmes naturels (Rabiou et al., 2016). Le Burkina Faso, de par sa position géographique, se trouve dans ces conditions d'instabilité écologique auxquelles s'ajoutent des pressions anthropiques (Kaboré et al., 2017). Ces pressions conduisent à la dégradation accélérée des formations végétales naturelles, entraînant la régression et la disparition de nombreuses espèces ligneuses utilisées par les populations locales (Traoré et al., 2019; Ganamé et al., 2020). Depuis la Conférence de Rio en 1992, la dégradation des ressources naturelles est affichée comme une préoccupation majeure car elle constitue une menace pour l'humanité. La mise en œuvre d'un programme de gestion forestière axé sur les espèces pourvoyeuses de PFNL, dans un contexte de variabilité climatique et d'anthropisation des habitats naturels, s'avère nécessaire et doit passer par une connaissance approfondie des espèces dans leur milieu naturel.

Au Burkina Faso, de nombreuses espèces ligneuses sont pourvoyeuses de PFNL et sont exploitées pour la transformation agroalimentaire (MEEVCC, 2017), notamment Adansonia digitata L., Balanites aegyptiaca (L.) Delile, Bombax costatum Pellegr. \& Vuillet, Parkia biglobosa (Jacq.) G. Don, Saba senegalensis (A. DC.) Pichon, Senegalia macrostachya (Rchb. ex DC.) Kyal. \& Boatwr., Tamarindus indica L., Vitellaria paradoxa C.F. Gaertn. et Ziziphus mauritiana Lam. Parmi ces espèces, certaines demeurent encore peu connues et sous-valorisées, notamment $S$. senegalensis.

Originaire d'Afrique subsaharienne, $S$. senegalensis (famille des Apocynaceae) est une liane ligneuse à latex blanc et à vrilles. Elle se rencontre dans les forêts, les savanes (photo 1), sur les plateaux, les collines rocheuses et les rives des cours d'eau. Elle est adaptée à différents types de sols et tolère les feux de végétation peu violents (Arbonnier, 2019). Toutes les parties de la plante sont utilisées par les populations locales pour des besoins divers (Ouédraogo et al., 2017 ; Traoré et al., 2019). Ses

fruits riches en vitamines et en protéines (Sarr et al., 2018) sont utilisés comme compléments alimentaires et font l'objet d'un commerce important (Lamien et al., 2018). Malgré son importance socio-économique, cette espèce n'a fait l'objet que de peu d'investigations. La plupart des études disponibles concernent sa biologie de reproduction (Traoré, 2000), les valeurs nutritionnelle et socio-économique de ses produits (Lamien et al., 2010 ; Sarr et al., 2018), la perception des populations locales sur sa vulnérabilité (Ouédraogo et al., 2017) et sa variabilité morphologique (Diouf et al., 2019). L'état des populations de l'espèce demeure mal connu. Au regard de cette situation et des pressions anthropiques en forte croissance, la présente étude s'intéresse à l'évaluation des caractéristiques démographiques de $S$. senegalensis suivant le gradient climatique afin d'orienter des stratégies adaptées pour en assurer une gestion rationnelle et durable au Burkina Faso. Les objectifs spécifiques de l'étude sont de déterminer la structure spatiale et décrire l'habitat de $S$. senegalensis, d'évaluer les paramètres structuraux de ses populations en fonction de la zone climatique et, enfin, d'estimer l'influence des variables environnementales sur la régénération de l'espèce.

\section{Matériel et méthodes}

\section{Zone d'étude}

L'étude a été menée dans trois provinces du Burkina Faso réparties sur trois zones climatiques que sont la zone sahélienne (Yatenga), la zone soudano-sahélienne (Mouhoun) et la zone soudanienne (Kénédougou) (Fontès et Guinko, 1995) (figure 1). Les conditions climatiques des zones d'étude sont marquées de façon générale par une alternance de deux saisons contrastées, une saison sèche et une saison pluvieuse. Toutefois, les paramètres climatiques et environnementaux varient en fonction des trois zones climatiques (tableau I). Les principales activités économiques des populations locales sont l'agriculture, l'élevage, le commerce, l'artisanat et l'exploitation des produits forestiers non ligneux.

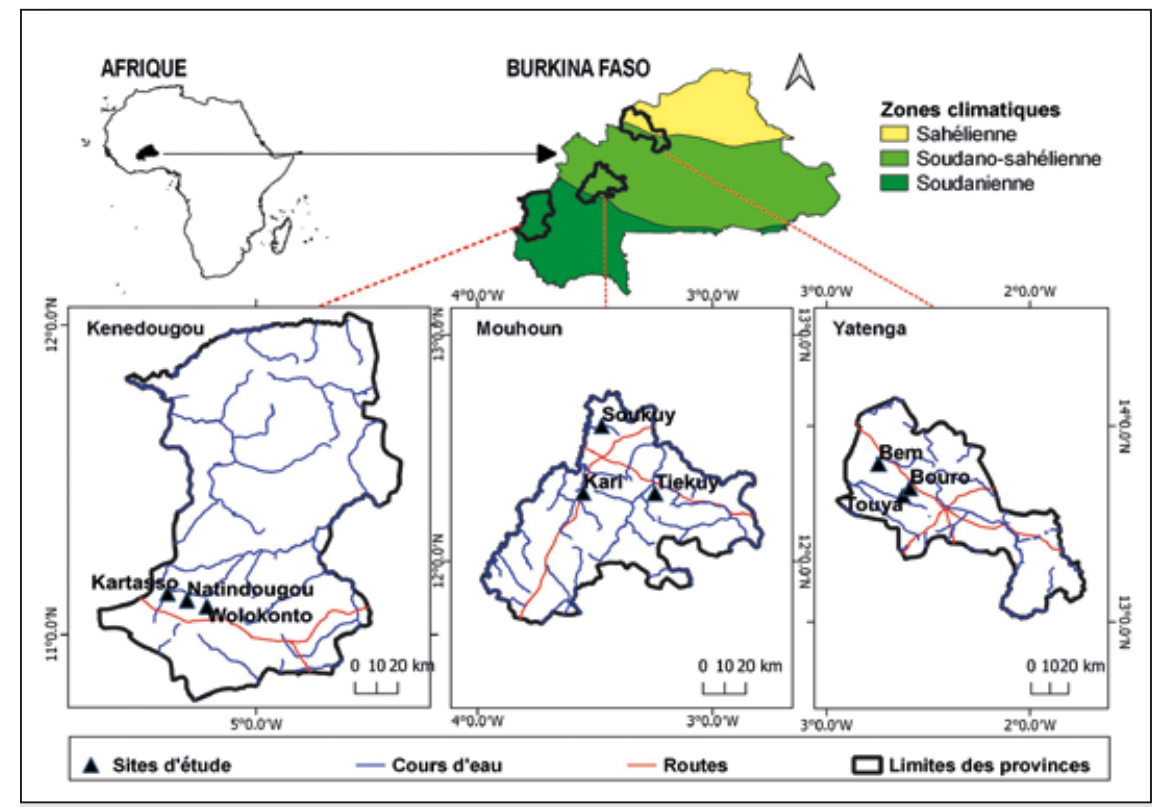

Figure 1.

Localisation de la zone d'étude. 
Tableau I.

Variations des paramètres environnementaux en fonction des zones climatiques.

\begin{tabular}{l|l|l|l}
$\begin{array}{l}\text { Paramètres } \\
\text { environnementaux }\end{array}$ & Sahélienne & $\begin{array}{c}\text { Zones climatiques } \\
\text { Soudano-sahélienne }\end{array}$ & Soudanienne \\
\hline Saison pluvieuse & Juin-septembre & Juin-octobre & Avril-octobre \\
\hline Pluviométrie & $\langle 600 \mathrm{~mm}$ & $600-900 \mathrm{~mm}$ & $>1000 \mathrm{~mm}$ \\
\hline Température & $30^{\circ} \mathrm{C}$ & $20-30^{\circ} \mathrm{C}$ & $20-25^{\circ} \mathrm{C}$ \\
\hline Réseau hydrographique & $\begin{array}{l}\text { Sources des rivières Sourou, } \\
\text { Nakambé }\end{array}$ & $\begin{array}{l}\text { Fleuve Mouhoun } \\
\text { et ses affluents }\end{array}$ & $\begin{array}{l}\text { Fleuve Comoé et son } \\
\text { affluent Léraba }\end{array}$ \\
\hline Types de sols & $\begin{array}{l}\text { Minéraux, ferrugineux, } \\
\text { hydromorphes }\end{array}$ & $\begin{array}{l}\text { Vertisols, ferrugineux, } \\
\text { hydromorphes }\end{array}$ & $\begin{array}{l}\text { Hydromorphes, } \\
\text { ferrallitiques }\end{array}$ \\
\hline Types de végétation & Savanes, steppes & Savanes, forêts-galeries & $\begin{array}{l}\text { Savanes, forêts claires, } \\
\text { forêts-galeries }\end{array}$
\end{tabular}

Variables climatiques (Fontès et Guinko, 1995) ; Réseau hydrographique (BUNASOLS, 2004 ; 2006) ; Végétation (Fontès et Guinko, 1995).

\section{Échantillonnage et collecte de données}

Dans chaque zone climatique, trois sites ont été retenus suivant un échantillonnage aléatoire (Kakaï et al., 2015), orienté sur la présence de Saba senegalensis. À la fin de la saison des pluies (octobre-novembre 2017), des inventaires forestiers ont été réalisés sur 10 placeaux de $50 \mathrm{~m} \times 20 \mathrm{~m}$ par site, soit 30 placeaux par zone climatique. Les données collectées ont concerné les paramètres dendrométriques, les coordonnées géographiques et les caractéristiques du milieu (type de végétation, topographie, taux de recouvrement des ligneux, texture du sol) de chaque placeau et les variables climatiques des sites. Les mesures des paramètres dendrométriques ont porté sur le diamètre de la tige au collet et à $1,30 \mathrm{~m}$ du sol (dbh) pour les sujets adultes ( $\mathrm{dbh} \geq$ $2 \mathrm{~cm}$ ) (Gerwing et al., 2006). L'état sanitaire de chaque individu adulte a été noté selon la codification suivante : $1=$ individu sain, 2 = individu malade (individu attaqué, parasité ou partiellement endommagé) et 3 = individu mort.

L'inventaire de la régénération naturelle a été effectué sur les mêmes placeaux de $50 \mathrm{~m}$ x 20 m. Il a concerné les individus juvéniles ( $\mathrm{dbh}<2 \mathrm{~cm}$ ) regroupés en plantules (hauteur $<0,5 \mathrm{~m}$ ) et en jeunes plants (hauteur $\geq 0,5 \mathrm{~m}$ ) (Gao et al., 2017). Les diamètres de la tige au collet et à $20 \mathrm{~cm}$ du sol ont été mesurés, respectivement chez les plantules et les jeunes plants, à l'aide d'un pied à coulisse (précision $0,01 \mathrm{~mm}$ ).

Pour les besoins d'évaluation de l'effet des précipitations et de la température sur la régénération naturelle de S. senegalensis, des données climatiques des sites ont été téléchargées à partir de WorldClim version 2 (Fick et Hijmans, 2017) avec une résolution spatiale de $30 \mathrm{~s}$ d'arc (environ $1 \mathrm{~km}$ à l'équateur).

\section{Analyse des données}

\section{Distribution spatiale et caractérisation des habitats de Saba senegalensis}

Pour apprécier la distribution spatiale de l'espèce, l'Indice de Green (IG) a été calculé à l'aide de la formule suivante (Jayaraman, 1999) :

$I G=\frac{\mathrm{IB}-1}{\mathrm{n}-1}($ équation 1$)$

avec $\mid B=\frac{\sigma^{2}}{N}$, indice de Blackman, où $\sigma^{2}$ et $N$ sont respectivement, la variance et la densité moyenne des individus adultes, et $\mathrm{n}$ le nombre total d'individus adultes dans le placeau. La valeur de l'IG varie entre 0 (distribution aléatoire) et 1 (distribution agrégative).

Les habitats naturels de l'espèce ont été caractérisés à partir des données de la topographie, du type de végétation et de la texture du sol.

\section{Caractéristiques dendrométriques et démographiques}

Les paramètres suivants ont été calculés pour décrire les caractéristiques dendrométriques et démographiques de l'espèce.

- La densité moyenne (Dm) des individus de S. senegalensis :

$D m=\frac{k}{s}$ (équation 2)

où k est le nombre total d'individus (adultes, jeunes plants ou plantules) dans le placeau et $\mathrm{s}$ la superficie du placeau ( $\mathrm{s}=0,1 \mathrm{ha}$ ).

- Le diamètre moyen (Dg) des individus, exprimé en $\mathrm{cm}$ pour les adultes et les jeunes plants et en $\mathrm{mm}$ pour les plantules : 
$D g=\left(\frac{1}{k} \sum_{i=1}^{k} d i^{2}\right)^{1 / 2}($ équation 3$)$

où k est défini comme dans l'équation (2) et di le diamètre de l'individu i. Pour un individu i, ramifié en $\mathrm{n}$ tiges en dessous du niveau de mesure, le diamètre quadratique a été calculé comme suit :

$D q=\left(\sum_{j=1}^{n} d s^{2} j\right)^{1 / 2}($ équation 4)

- La surface terrière des individus adultes par placeau (G, en $\mathrm{m}^{2} / \mathrm{ha}$ )

$G=\frac{\pi}{4 s} \sum_{i=1}^{k} 10^{-4} \mathrm{di}^{2}$ (équation 5)

avec di le diamètre de l'individu i du placeau, $s=0,10$ ha.

Le test de comparaison non paramétrique de Wilcoxon au seuil de $5 \%$ a été utilisé pour comparer les valeurs des paramètres calculés entre les zones climatiques. Les analyses statistiques ont été réalisées à l'aide du logiciel $R$ version 3.6.0 (R Core Team, 2019).

\section{Structure des populations de Saba senegalensis}

La structure des populations de S. senegalensis a été analysée grâce à la distribution des individus en classes de diamètre d'amplitude $3 \mathrm{~cm}$. Les structures ont été ajustées par une surimposition de la distribution théorique de Weibull à trois paramètres (Husch et al., 2003) à l'aide du logiciel Minitab14. La fonction de probabilité de Weibull (f) est exprimée selon la formule :

$f(x)=\frac{c}{b}\left(\frac{x-a}{b}\right)^{c-1} \exp \left\{-\left(\frac{x-a}{b}\right) c\right\}$ (équation 6)

avec $: \mathrm{x}=$ diamètre de la tige $; \mathrm{a}=$ paramètre de seuil $; \mathrm{b}=$ paramètre de taille ; $c=$ paramètre de forme. Un test d'ajustement basé sur une analyse log-linéaire a été effectué pour assurer un ajustement approprié de la structure observée à la distribution théorique à l'aide du logiciel SAS version
10.2. Les structures en classes de diamètre ajustées à la distribution de Weibull à deux paramètres (le paramètre « $a$ » étant nul) ont été analysées pour cerner la tendance démographique de la régénération naturelle.

\section{Paramètres affectant la régénération de Saba senegalensis}

L'analyse des variables climatiques a concerné la température et les précipitations. Pour tester l'effet de ces deux variables climatiques et du taux de recouvrement des ligneux sur la régénération, le modèle linéaire généralisé (GLM) (Crawley, 2007) a été utilisé avec une distribution de Poisson à un seuil de significativité de $5 \%$ à l'aide du logiciel R version 3.6.0 (R Core Team, 2019).

\section{Résultats}

\section{Distribution spatiale et caractéristiques des habitats de Saba senegalensis}

Les valeurs de l'indice de Green indiquent une répartition spatiale agrégative des individus de $S$. senegalensis dans les populations. Cette répartition agrégative est plus prononcée dans la zone soudano-sahélienne que dans les deux autres zones climatiques (tableau II). Les données situationnelles des habitats naturels de S. senegalensis ont révélé que l'espèce colonise une large gamme de milieux avec des taux d'occurrence variables (tableau II).

\section{Caractéristiques démographiques des populations de Saba senegalensis}

Les populations de $S$. senegalensis révèlent une densité globale moyenne de 48,1 individus adultes à l'hectare. Les variations entre les zones climatiques sont significatives ( $p<0,05)$, avec la densité la plus faible observée dans la zone soudano-sahélienne (tableau III). Les valeurs de la surface terrière sont comparables entre les zones sahélienne et soudano-sahélienne mais significativement plus faibles dans la zone soudanienne. Pour l'état sanitaire, le taux le

Tableau II.

Caractéristiques de la distribution spatiale et des habitats de Saba senegalensis.

\begin{abstract}
Paramètres de la distribution des individus et caractéristiques des habitats

\section{vidus}

Indice de Green Occurrence de $S$. senegalensis en fonction des types d'occupation des terres (\%)

Occurrence de S. senegalensis en fonction des types de texture des sols des habitats (\%)

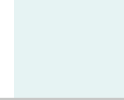

Savane

Forêt

Jachère

Champ

Sableux

Argileux

Limoneux

Gravillonnaire
Zones climatiques Soudano-sahélienne
Soudanienne

0,43

33,33

10,00

20,00

36,67

10,00

16,66

46,66

26,67
0,28

38,10

42,86

11,90

7,14

4,76

11,90

35,71

47,62 
Tableau III.

Paramètres démographiques et structuraux (moyenne \pm écart-type) de Saba senegalensis en fonction des zones climatiques.

\begin{tabular}{|c|c|c|c|c|}
\hline $\begin{array}{l}\text { Paramètres structuraux } \\
\text { et démographiques }\end{array}$ & Sahélienne & $\begin{array}{r}\text { Zones climatiques } \\
\text { Soudano-sahélienne }\end{array}$ & Soudanienne & $p$-value \\
\hline $\begin{array}{l}\text { Adultes } \\
\text { Densité (individus/ha) }\end{array}$ & $65,3 \pm 53,5^{b}$ & $22,2 \pm 24,2^{a}$ & $56,8 \pm 57,9^{b}$ & $<0,001$ \\
\hline $\mathrm{Dbh}(\mathrm{cm})$ & $11,4 \pm 5,9^{a}$ & $19,9 \pm 11,6^{b}$ & $9,0 \pm 4,4^{a}$ & 0,04 \\
\hline Surface terrière (m²/ha) & $0,5 \pm 0,45^{b}$ & $0,6 \pm 0,7^{b}$ & $0,30 \pm 0,3^{a}$ & $<0,001$ \\
\hline individus sains (\%) & $28,8 \pm 33,1^{a}$ & $54,3 \pm 47^{b}$ & $53,2 \pm 36,3^{b}$ & 0,01 \\
\hline $\begin{array}{l}\text { Jeunes plants } \\
\text { Densité (individus/ha) }\end{array}$ & $27,2 \pm 23,3^{a}$ & $42 \pm 26,8^{a}$ & $100,6 \pm 91,4^{b}$ & $<0,001$ \\
\hline Diamètre à $20 \mathrm{~cm}$ du sol $(\mathrm{cm})$ & $0,8 \pm 0,6^{b}$ & $3,80 \pm 3,7^{a}$ & $0,7 \pm 0,6^{b}$ & $<0,001$ \\
\hline $\begin{array}{l}\text { Plantules } \\
\text { Densité (individus/ha) }\end{array}$ & $609 \pm 575,5^{a}$ & $1093,3 \pm 1124,8^{b}$ & $573,9 \pm 456,1^{a}$ & 0,01 \\
\hline Diamètre au collet $(\mathrm{cm})$ & $0,4 \pm 1,1^{\mathrm{ab}}$ & $0,3 \pm 0,2^{\mathrm{a}}$ & $0,4 \pm 0,2^{b}$ & $<0,001$ \\
\hline
\end{tabular}

plus élevé de pieds sains de $S$. senegalensis a été observé dans la zone soudano-sahélienne, taux comparable à celui de la zone soudanienne (tableau III).

En ce qui concerne la régénération, la densité moyenne globale des jeunes plants de $S$. senegalensis est de 57 individus à l'hectare. La variation en fonction des zones climatiques révèle une densité significativement plus élevée dans la zone soudanienne (tableau III). La densité des plantules montre un potentiel de régénération élevé avec une valeur moyenne globale de 759 individus à l'hectare. La densité des plantules est significativement plus élevée dans la zone soudano-sahélienne (tableau III).

\section{Structure en classes de diamètre}

Dans chacune des trois zones climatiques, la structure de la distribution des individus adultes en classes de diamètre présente une allure en J-renversée (figure 2). La valeur du paramètre de forme " $c$ » de la distribution de Weibull comprise entre 1 et 3,6 est caractéristique d'une population à prédominance d'individus jeunes. Toutefois, il apparaît une irrégularité de la succession des effectifs dans les classes de diamètre, surtout marquée dans la zone soudano-sahélienne (figure 2). L'analyse log-linéaire montre que la distribution observée s'ajuste bien à la distribution théorique de Weibull.

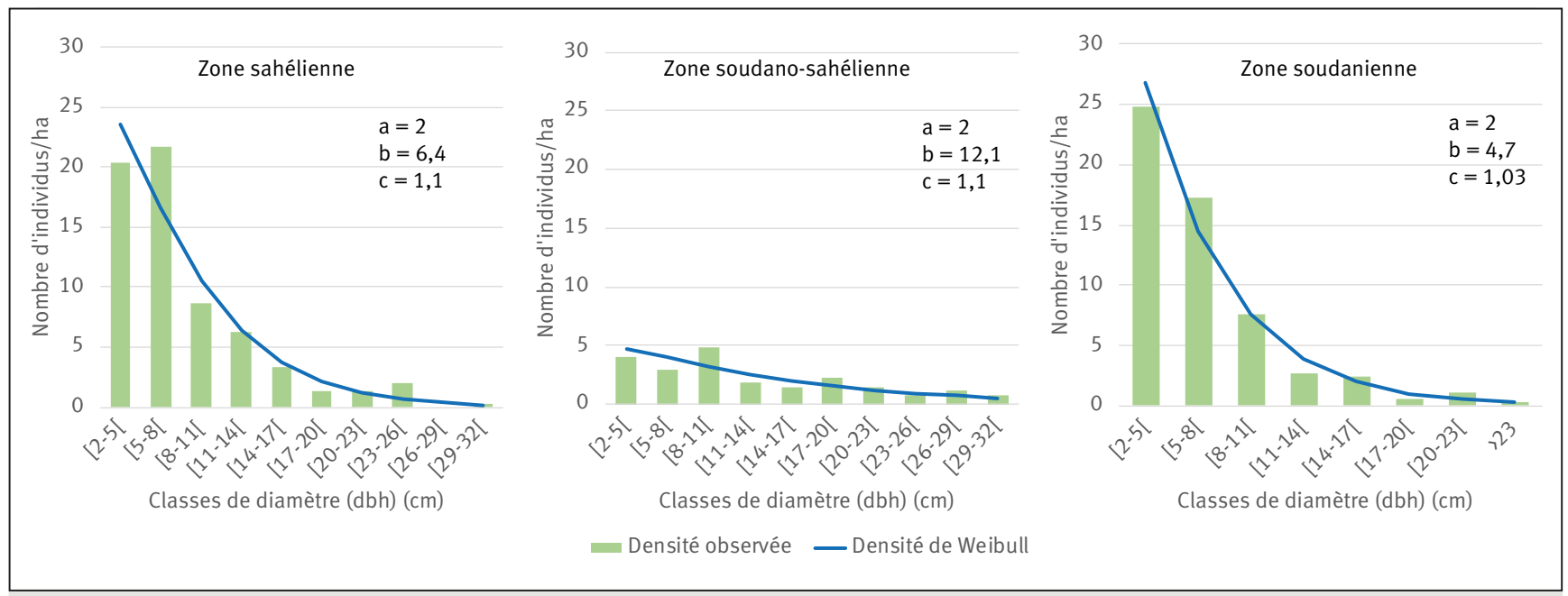

Figure 2.

Structure de la distribution en classes de diamètre ( $\mathrm{Dbh}$ ) des individus adultes de Saba senegalensis dans les trois zones climatiques. $a$ = paramètre de seuil ; $b=$ paramètre de taille ; $c=$ paramètre de forme. 


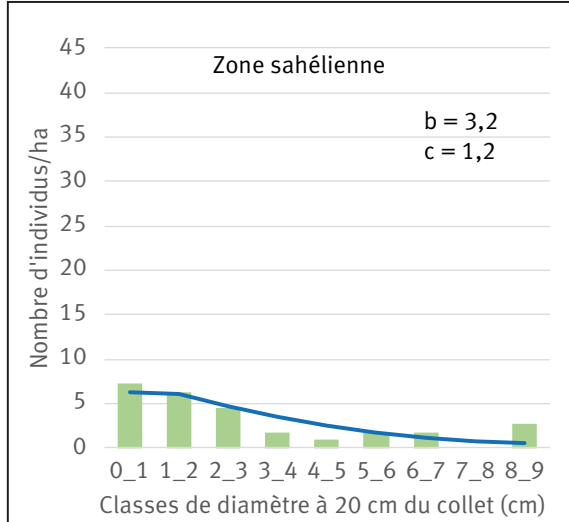

Classes de diamètre à $20 \mathrm{~cm}$ du collet $(\mathrm{cm})$

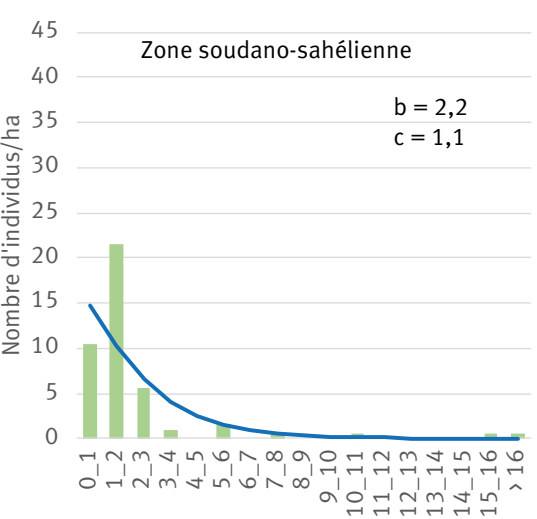

Classes de diamètre à $20 \mathrm{~cm}$ du collet $(\mathrm{cm})$

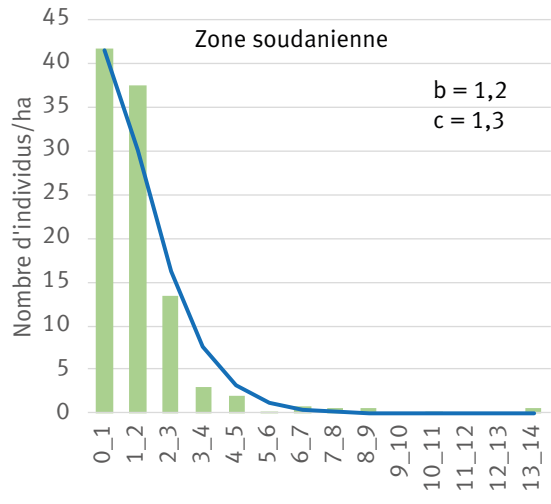

Classes de diamètre à $20 \mathrm{~cm}$ du collet $(\mathrm{cm})$

Densité observée —Densité de Weibull

Figure 3.

Structure de la distribution en classes de diamètre (à $20 \mathrm{~cm}$ du collet) des jeunes plants de Saba senegalensis dans les trois zones climatiques. $b=$ paramètre de taille $; c=$ paramètre de forme.

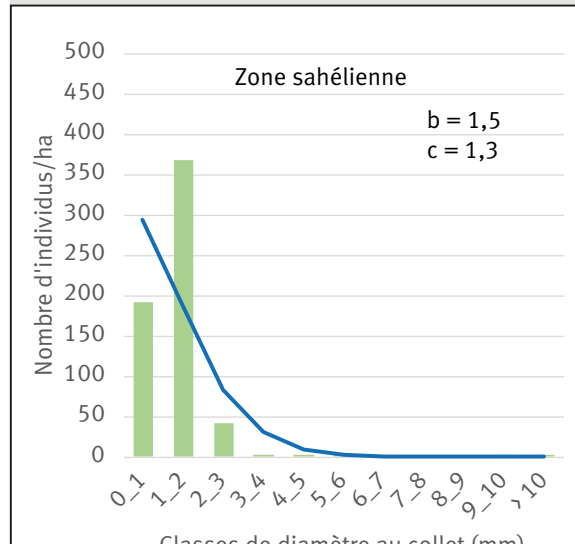

Classes de diamètre au collet $(\mathrm{mm})$

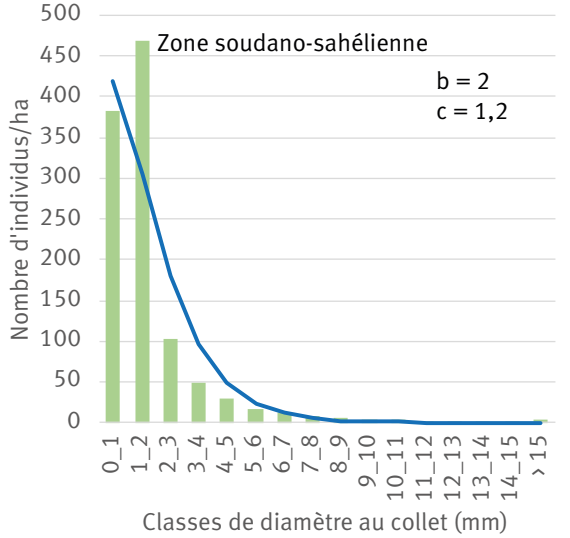

Classes de diamètre au collet $(\mathrm{mm})$

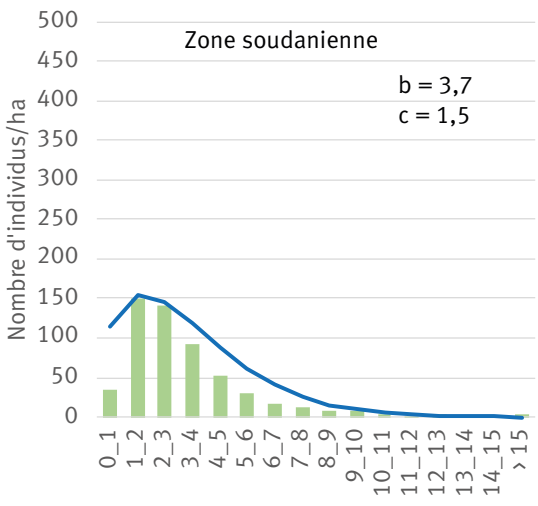

Classes de diamètre au collet $(\mathrm{mm})$

Densité observée _ Densité de Weibull

\section{Figure 4.}

Structure de la distribution en classes de diamètre (au collet) des plantules de Saba senegalensis dans les trois zones climatiques. $b=$ paramètre de taille ; $c=$ paramètre de forme.

Les structures démographiques de la strate de la régénération montrent aussi une allure en forme de J-renversée, traduisant des effectifs plus élevés dans les petites classes de diamètre des jeunes plants (figure 3). Cette allure est caractéristique d'un bon potentiel de renouvellement des populations. Cependant, la baisse drastique des effectifs dans les classes de plus de $3 \mathrm{~cm}$ de diamètre révèle sans doute des difficultés de survie des jeunes plants.

Les structures en classes de diamètre au collet des plantules montrent une allure en forme de J-renversée avec une valeur du paramètre " $c$ » de Weibull comprise entre 1 et 3,6. Les petites classes (0-4 mm) contiennent les effectifs les plus élevés, indiquant un fort potentiel de recrutement dans les populations. Toutefois, la chute brutale des effectifs des petites classes vers les grandes classes de diamètre révèle des difficultés de survie des plantules. Dans la zone sahélienne, l'effectif de plantules de diamètre supérieur à $4 \mathrm{~mm}$ est quasi nul (figure 4).

\section{Paramètres environnementaux affectant la régénération de Saba senegalensis}

Le modèle linéaire généralisé (GLM) correspond à une équation de la forme :

$\ln (Y)=\beta_{0}+\beta_{1}$ précipitations $+\beta_{2}$ taux de recouvrement + $\beta_{3 \mathrm{t}}$ empérature

avec $\beta_{0}=$ intercepte (constante) ; $\beta_{1} \beta_{2}$ et $\beta_{3}$ des estimateurs du modèle.

Ce modèle indique que les valeurs élevées de la température et des précipitations contribuent de manière positive à l'établissement de la régénération de $S$. senegalensis ( $z>0)$. En revanche, une valeur élevée du taux de recouvrement qui représente la proportion de la couverture végétale dans les placeaux ne favorise pas une forte densité de la régénération de $S$. senegalensis ( $\mathrm{z}<0$ ) (tableau IV). En effet, les faibles densités de régénération de $S$. senegalensis s'observent dans les milieux très boisés. 
Tableau IV.

Niveau d'influence des paramètres environnementaux sur la régénération de Saba senegalensis.

\begin{tabular}{l|r|r|r|r}
\hline Variables & Estimateur & Erreur standard & Z value & $\operatorname{Pr}(>|\mathbf{Z}|)$ \\
\hline Intercepte & $-1,021 \mathrm{E}+01$ & $2,673 \mathrm{E}-01$ & $-38,19$ & $<0,001$ \\
\hline Précipitations & $2,526 \mathrm{E}-03$ & $4,411 \mathrm{E}-05$ & 57,26 & $<0,001$ \\
\hline Taux de recouvrement & $-2,407 \mathrm{E}-03$ & $2,222 \mathrm{E}-04$ & $-10,83$ & $<0,001$ \\
\hline Température & $5,338 \mathrm{E}-02$ & $8,338 \mathrm{E}-04$ & 64,03 & $<0,001$ \\
\hline
\end{tabular}

\section{Discussion}

\section{Distribution spatiale et habitats de Saba senegalensis}

La tendance agrégative des populations naturelles de S. senegalensis dans les trois zones climatiques traduit la capacité de l'espèce à coloniser son environnement grâce à ses aptitudes au drageonnage et au rejet de souche, comme cela a été rapporté pour Isoberlinia doka (Bationo et al., 2005). En effet, le drageonnage est une stratégie de reproduction asexuée qui favorise la distribution grégaire des peuplements (Bellefontaine et al., 2000). Ce mécanisme de reproduction est développé par certaines espèces pour compenser les limites de la reproduction sexuée dans les milieux perturbés (Bellefontaine et al., 2000 ; Bationo et al., 2005). Le drageonnage constitue une stratégie alternative pour la régénération naturelle des espèces de savane qui sont annuellement confrontées à la sécheresse et aux feux de végétation, face auxquels les semis résistent peu (Bognounou et al., 2010 ; Ouédraogo et Thiombiano, 2012).

\section{Paramètres structuraux des populations de Saba senegalensis}

La faible densité des individus adultes de $S$. senegalensis des populations de la zone soudano-sahélienne pourrait s'expliquer par la forte pression anthropique à travers la coupe et les défriches agricoles (photos 2). Ces pratiques contribuent à la dégradation des formations végétales, conduisant à la fragmentation des habitats naturels. Les résultats de la présente étude corroborent ceux de Zampaligre et al. (2019) et de Abdourhamane et al. (2017) qui ont montré que la pression anthropique réduit la densité des individus adultes dans les populations végétales.

Les valeurs faibles du diamètre moyen et de la surface terrière des individus de $S$. senegalensis dans les zones sahélienne et soudanienne montrent qu'il y a peu d'individus âgés. La surexploitation à laquelle $S$. senegalensis est soumise et qui cause sa vulnérabilité (Ouédraogo et al., 2017) touche principalement les sujets adultes et peut aussi expliquer les faibles valeurs des diamètres moyens et de la surface terrière obtenues dans les zones sahélienne et soudanienne. En effet, plusieurs auteurs tels que Gaoué et Ticktin (2010) ont montré que l'exploitation irrationnelle des fruits et des branches des ligneux est susceptible d'affecter négativement leur surface terrière.

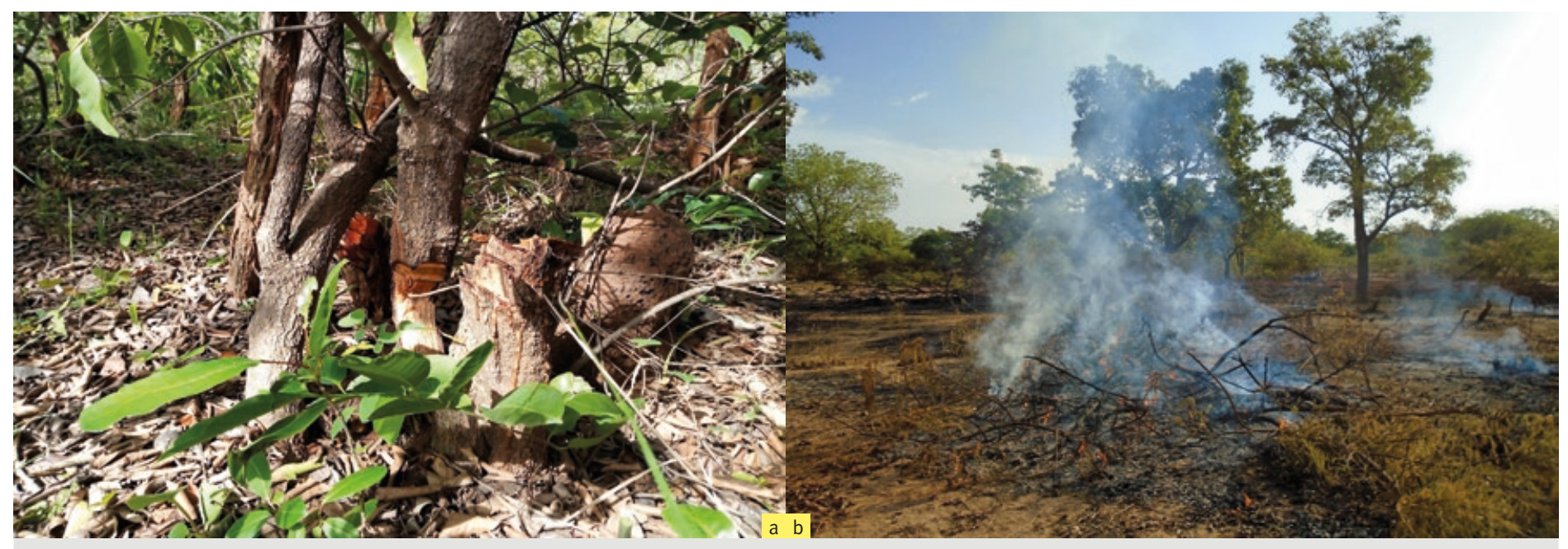

Photos 2.

Manifestations de la pression anthropique à travers la coupe des sujets adultes

(a) et les défriches-brûlis (b) dans les peuplements de Saba senegalensis en zone soudano-sahélienne.

Photos B. Kabré. 
La faible densité des jeunes plants observée dans la zone sahélienne révèle l'effet des facteurs de perturbation (surpâturage, feux de végétation et sécheresses récurrentes) sur les populations de $S$. senegalensis, tout comme cela a été rapporté pour d'autres espèces ligneuses en zone semi-aride (Bognounou et al., 2010). De même, les plantules résistent peu aux facteurs de dégradation du milieu. La plus faible densité des plantules a été observée dans la zone soudanienne qui est pourtant la plus humide du Burkina Faso. Les inondations causées par la forte pluviosité de cette zone pourraient être un facteur limitant de la régénération de $S$. senegalensis. Les résultats de cette étude corroborent ceux de Assédé et al. (2015) qui ont montré que les inondations compromettent le développement de la strate juvénile des ligneux après la germination. La forte densité des sujets adultes de $S$. senegalensis dans certaines populations pourrait conduire à une intense compétition intra-spécifique pour les éléments nutritifs et être également source d'une faible régénération naturelle. Cela a été rapporté par Atanasso et al. (2019) qui montrent que la densité de la strate juvénile est négativement influencée par celle des adultes.

\section{État des populations de Saba senegalensis}

Dans les trois zones climatiques, la distribution en classes de diamètre des individus adultes de $S$. senegalensis révèle globalement une stabilité des populations. L'abondance des individus dans les classes de petit diamètre traduit un potentiel de renouvellement des populations. Cependant, l'irrégularité de la succession des effectifs dans les classes de diamètre révèle l'effet des perturbations qui affectent la survie et le bon développement des individus. Cela est dû aux pressions humaines à travers la pratique de l'agriculture et le prélèvement des différentes parties des individus adultes de $S$. senegalensis. En effet, ces prélèvements par les populations locales pour leurs besoins en pharmacopée, en énergie et en artisanat (Ouédraogo et al., 2017 ; Traoré et al., 2019) constituent une menace pour la survie de cette espèce. L'exploitation des différentes parties de l'arbre se manifeste souvent par des dégâts de diverses formes généralement observés sur les tiges et qui conduisent à la mort des individus (Zampaligré et al., 2019).

Pour les jeunes plants de $S$. senegalensis, l'irrégularité de la succession des effectifs entre les classes de diamètre montre que les individus rencontrent des problèmes de survie dans les trois zones climatiques. Ces difficultés sont relatives aux perturbations des habitats par les activités agricoles, les feux et le pâturage (Sewade et al., 2016 ; Assogbadjo et al., 2017).

Concernant les plantules de $S$. senegalensis, l'abondance des individus dans les petites classes de diamètre dans les trois zones climatiques révèle un important potentiel de régénération (Lankoandé et al., 2019 ; Ganamé et al., 2020) tributaire de sa grande reproduction fruitière. La baisse brutale des effectifs dans les classes de diamètre supérieur à $4 \mathrm{~mm}$ dans la zone sahélienne est probablement due au stress hydrique (Kozlowski, 2002).

\section{Paramètres environnementaux affectant la régénération de Saba senegalensis}

Contrairement à la température et aux précipitations, l'augmentation du taux de recouvrement des ligneux influence négativement le potentiel de régénération de S. senegalensis. Ces résultats sont similaires à ceux de Gao et al. (2017) qui ont souligné que le taux de recouvrement élevé réduit la densité de la régénération des ligneux. L'ombrage est un facteur limitant pour la croissance des plantules de S. senegalensis qui est une plante héliophile (Traoré, 2000). Certaines espèces d'herbacées et d'arbustes sont adaptées à ces conditions de luminosité réduites alors que les lianes s’y développent mal (Hallé et al., 1978). La température et la pluviométrie, en intervenant dans la nutrition minérale et l'activité photosynthétique de la plante (Bloesch et al., 2009), influencent l'établissement de la strate juvénile de $S$. senegalensis.

\section{Conclusion}

Cette étude a montré que les populations de Saba senegalensis présentent une distribution agrégative avec des structures relativement stables dans les trois zones climatiques. Cependant, la faiblesse du taux d'individus sains dans les populations de la zone sahélienne et de la densité des individus adultes dans la zone soudano-sahélienne compromet la disponibilité de la ressource. L'espèce présente un potentiel de régénération élevé dans les trois zones climatiques. Bien que $S$. senegalensis montre une certaine résilience à travers ses populations stables et une bonne capacité de recrutement, elle demeure vulnérable au regard des difficultés apparentes d'affranchissement des plantules. L'effet combiné de la péjoration climatique et de la pression anthropique compromet la conservation de la plante dans son habitat naturel. Cette situation révèle l'urgence de la mise en place d'une planification de sa gestion durable telle que son intégration dans les programmes de domestication des plantes locales.

\section{Remerciements}

Les auteurs de ce présent article remercient le projet TreeFood $n^{\circ}$ 1507-143 d'Agropolis Fondation pour le soutien financier qui a permis la conduite de cette étude. Ils expriment également leur reconnaissance aux populations locales des provinces du Yatenga, du Mouhoun et du Kénédougou pour leur collaboration. 


\section{Références}

Abdourhamane H., Rabiou H., Diouf A., Morou B., Mahamane A., Bellefontaine R., 2017. Structure démographique et répartition spatiale des populations de Sclerocarya birrea (A. Rich.) Hochst. du secteur sahélien du Niger. Bois et Forêts des Tropiques, 333 (3) : 55-66. https://revues.cirad. $\mathrm{fr} /$ index.php/BFT/article/view/ID-BFT-161121

Arbonnier M., 2019. Arbres, arbustes et lianes des zones sèches d'Afrique de l'Ouest. Versailles, France, Éditions Quæ, 776 p.

Assédé E. S. P., Azihou F. A., Adomou A. C., Oumoro M., Sinsin B., 2015. Effet du relief sur la régénération des espèces ligneuses en zone soudanienne du Bénin. Bois et Forêts des Tropiques, 326 (4) : 15-24. https://doi.org/10.19182/ bft2015.326.a31280

Assogbadjo A. E., Glèlè Kakaï R., Vodouhê F. G., Djagoun C. A. M. S., Codjia J. T. C., Sinsin B., 2012. Biodiversity and socioeconomic factors supporting farmers' choice of wild edible trees in the agroforestry systems of Benin (West Africa). Forest Policy and Economics, 14: 41-49. http://doi. org/10.1016/j.forpol.2011.07.013

Assogbadjo A. E., Mensah S., Kakaï R. G., 2017. The relative importance of climatic gradient versus human disturbance in determining population structure of Afzelia africana in the Republic of Benin. Southern Forests: a Journal of Forest Science, 79: 125-132. http://dx.doi.org/10.2989/2070262 0.2016 .1255406

Atanasso J. A., Mensah S., Azihou A. F., Djossa B. A., Kakaï R. G., Assogbadjo A. E., 2019. Heterospecific Tree Density and Environmental Factors Affect Afzelia africana Sm. Population Structure in the Pendjari Biosphere Reserve, West Africa: Implications for Management and Restoration. Tropical Conservation Science, 12: 1-12. http://doi. org/10.1177/1940082918822604

Bationo B. A., Ouédraogo S. J., Somé A. N., Pallo F., Boussim I. J., 2005. Régénération naturelle d'Isoberlinia doka Craib. et Stapf. dans la forêt classée du Nazinon (Burkina Faso). Cahiers Agricultures, 14 (3) : 297-304. https://revues.cirad. $\mathrm{fr} /$ index.php/cahiers-agricultures/article/view/30522

Bellefontaine R., Edelin C., Ichaou A., du Laurens D., Monsarrat A., Loquai C., 2000. Le drageonnage, alternative aux semis et aux plantations de ligneux dans les zones semiarides : protocole de recherches. Sécheresse, 11 : 221-226. Bloesch B., Viret O., Fabre A. L., Spring J. L., 2009. Évolution climatique et phénologie de la vigne de 1958 à nos jours. Revue Suisse de Viticulture, d'Arboriculture et d'Horticulture, 41 (3) : 143-149. https://www.revuevitiarbohorti.ch/ wp-content/uploads/2009 03 f 734.pdf

Bognounou F., Tigabu M., Savadogo P., Thiombiano A., Boussim I. J., Oden P. C., et al., 2010. Regeneration of five Combretaceae species along a latitudinal gradient in Sahelo-Sudanian zone of Burkina Faso. Annals of Forest Science, 67 (306): 1-10. https://doi.org/10.1051/forest/2009119
BUNASOLS (Bureau national des sols), 2004. Étude morpho-pédologique des provinces du Yatenga, du Loroum et du Zandoma. Ouagadougou, Burkina Faso, BUNASOLS, Rapport technique $\mathrm{n}^{\circ} 2,142 \mathrm{p}$.

BUNASOLS (Bureau national des sols), 2006. Étude morpho-pédologique des provinces du Mouhoun et des Balés. Ouagadougou, Burkina Faso, BUNASOLS, Rapport technique $\mathrm{n}^{\circ} 135,82 \mathrm{p}$.

Crawley M. J., 2007. The R Book. Chichester, United Kingdom, John Wiley, 940 p.

Diouf P., Diedhiou S., Goudiaby A. O. K., Ndoye I., Koïta B., 2019. Étude de la variabilité morphologique de Saba senegalensis (A. DC.) Pichon en Casamance (Sénégal). European Scientific Journal, 15 (9): 500-517. http://dx.doi. org/10.19044/esj.2019.v15n9p500

Fick S. E., Hijmans R. J., 2017. Worldclim 2: New 1-km spatial resolution climate surfaces for global land areas. International Journal of Climatology, 37 (12): 4302-4315. https://doi. org/10.1002/joc.5086

Fontès J., Guinko S., 1995. Carte de végétation et d'occupation des sols du Burkina Faso. Notice explicative. Toulouse, France, ministère de la Coopération français, projet Campus, $68 \mathrm{p}$.

Ganamé M., Bayen P., Ouédraogo I., Dimobe K., Thiombiano A., 2020. Woody species composition, diversity and vegetation structure of two protected areas along a climatic gradient in Burkina Faso (West Africa). Journal of the Institute of Botany, Academy of Sciences of the Czech Republic, 54: 163-175. https://doi.org/10.1007/s12224-019-09340-9

Gao W. Q., Ni Y. Y., Xue Z. M., Wang X. F., Kang F. F., Hu J., et al., 2017. Population structure and regeneration dynamics of Quercus variabilis along latitudinal and longitudinal gradients. Ecosphere, 8 (4): 1-15. https://doi.org/10.1002/ ecs2.1737

Gaoué O. G., Ticktin T., 2010. Effects of harvest of non-timber forest products and ecological differences between sites on the demography of African mahogany. Conservation Biology, 24 (2): 605-614. https://doi.org/10.1111/j.15231739.2009.01345.x

Gerwing J. J., Schnitzer S. A., Burnham R. J., Bongers F., Chave J., Dewalt S. J., et al., 2006. A standard Protocol for Liana Censuses. Biotropica, 38 (2): 256-261. https://doi. org/10.1111/i.1744-7429.2006.00134.x

Hallé F., Oldeman R. A. A., Tomlinson P. B., 1978. Tropical trees and forests: An architectural analysis. Springer-Verlag, $441 \mathrm{p}$.

Husch B., Beers T. W., Kershaw J. A. Jr., 2003. Forest Mensuration. $4^{\text {th }}$ Ed. Hoboken, NJ, USA, John Wiley, 343 p.

Jayaraman K., 1999. A statistical manual for forestry research. Bangkok, Thailand, FAO, 231 p.

Kaboré P. N., Ouédraogo A., Sanon M., Yaka P., Somé L., 2017. Caractérisation de la variabilité climatique dans la région du Centre-Nord du Burkina Faso entre 1961 et 2015. Climatologie, 14 : 82-95. http://dx.doi.org/10.4267/climatologie. 1268 
Kakaï R. G., Salako V. K., Lykke A. M., 2015. Techniques d'échantillonnage en études de la végétation. Annales des Sciences Agronomiques, 19 : 1-14.

Kozlowski T. T., 2002. Physiological ecology of natural regeneration of harvested and disturbed forest stands: implications for forest management. Forest Ecology and Management, 158: 195-221. https://doi.org/10.1016/ S0378-1127(00)00712-X

Lamien N., Ouédraogo S. J., Sanogo D., Kouyate A. M., Tougiani A., Vognan G., et al., 2018. Catalogue régional des arbres et arbustes alimentaires des terroirs sahéliens et soudaniens d'Afrique de l'Ouest : vers une meilleure valorisation de leurs potentiels nutritionnels. Bamako, Mali, INSAH, $79 \mathrm{p.}$

Lamien N., Traoré S., Kini F., 2010. Potentialités productive, nutritive et économique de la liane goïne (Saba sene-

galensis A. DC. Pichon) dans le Sahel Burkinabè. Études et Recherches Sahéliennes, 14-15 : 115-127.

Lankoandé B., Lykke A. M., Boussim J. I., Ouédraogo A., 2019. Structure, plant diversity and future management of Pentadesma butyracea stands, an endangered oil tree species in Burkina Faso, West Africa. Forestry, 92 (5): 591-598. https://doi.org/10.1093/forestry/cpz034

Lykke A. M., Padonou E. A., 2019. Carbohydrates, proteins, fats and other essential components of food formative trees in West Africa. Heliyon, 5 (5): e01744. https://doi. org/10.1016/j.heliyon.2019.e01744

MEEVCC (Ministère de l'Environnement, de l'Économie Verte et du Changement Climatique), 2017. Étude sur les aspects socioéconomiques des filières prioritaires des produits forestiers non ligneux : Adansonia digitata L., Balanites aegyptiaca (L.) Delile, Bombax costatum Pellegr. \& Vuillet, Parkia biglobosa (Jacq.) G. Don, Saba senegalensis (A. DC.) Pichon, Senegalia macrostachya (Rchb. ex DC.) Kyal. \& Boatwr., Tamarindus indica L., Vitellaria paradoxa C.F. Gaertn. et Ziziphus mauritiana Lam. Rapport final, Burkina Fao, $81 \mathrm{p}$.

Ouédraogo A., Thiombiano A., 2012. Regeneration pattern of four threatened tree species in Sudanian savannas of Burkina Faso. Agroforestry Systems, 86: 35-48. https://doi. org/10.1007/s10457-012-9505-9

Ouédraogo P., Bationo B. A., Sanou J., Traoré S., Barry S., Dayamba S. D., et al., 2017. Uses and vulnerability of ligneous species exploited by local population of northern Burkina Faso in their adaptation strategies to changing environments. Agriculture and Food Security, 6 (15): 1-16. https://doi.org/10.1186/s40066-017-0090-z

Rabiou H., Dan Guimbo I., Bationo A. B., Issaharou-Matchi I., Mahamane A., 2016. État des populations naturelles de Vitellaria paradoxa C.F. Gaertn. dans la zone soudanienne du Niger et du Burkina Faso (Afrique de l'Ouest). Revue Ivoirienne des Sciences et Technologie, $28: 428-441$. https:// revist.net/REVIST 28/REVIST 28 26.pdf

$\mathrm{R}$ Core Team, 2019. A language and environment for statistical computing. Vienna, Austria, R Foundation for Statistical Computing. http://www.R-project.org
Sarr M. G., Ayessou N. C. M., Cissé M., Mar C., Sakho M., 2018. Optimisation de la pasteurisation du nectar de Saba senegalensis. International Journal of Innovation and Scientific Research, 39 (2) : 240-251. http://www.ijisr.issr-journals.org/abstract.php?article=IJISR-18-227-04

Sewade C., Azihou A. F., Fandohan A. B., Houehanou T. D., Houinato M., 2016. Diversité, priorité pastorale et de conservation des ligneux fourragers des terres de parcours en zone soudano-guinéenne du Bénin. Biotechnologie, Agronomie, Société et Environnement, 20 (2) : 113-129. https://doi. org/10.25518/1780-4507.12823

Traoré A., 2000. Reproduction et développement de Saba senegalensis (A.DC.) Pichon au Sénégal. Thèse de doctorat, Université Cheick Anta Diop, Sénégal, 200 p.

Traoré G. H., Sanou L., Koala J., 2019. Diversité d’utilisations et de connaissances des espèces locales préférées dans le corridor forestier de la Boucle du Mouhoun, Burkina Faso. Science et Technique, Sciences Naturelles et Appliquées, 38 (1) : 101-117.

Vodouhê F., Coulibaly O., Greene C., Sinsin B., 2009. Estimating the Local Value of Non-Timber Forest Products to Pendjari Biosphere Reserve Dwellers in Benin. Economic Botany, 63: 397-412. https://doi.org/10.1007/s12231. 009-9102-7

Zampaligré N., Kagambega W. F., Sanou L., Sawadogo L., 2019. Impact of Grazing Intensity on Floristic Diversity and Woody Structure in Grazing Area Near Kaboré Tambi National Park (Burkina Faso). Journal of Agriculture and Environmental Sciences, 8 (2): 106-115. https://doi.org/10.15640/ jaes.v8n2a13

\begin{tabular}{|c|c|}
\hline Rôle du contributeur & Noms des auteurs \\
\hline Analyse formelle & B. Kabré \\
\hline $\begin{array}{l}\text { Enquête et } \\
\text { investigation }\end{array}$ & B. Kabré \\
\hline Méthodologie & $\begin{array}{l}\text { B. Kabré, M. Belem/Ouédraogo, } \\
\text { B. Lankoandé, A. Ouédraogo }\end{array}$ \\
\hline Gestion de projet & A. Ouédraogo \\
\hline Supervision & M. Belem/Ouédraogo, A. Ouédraogo \\
\hline Validation & $\begin{array}{l}\text { M. Belem/Ouédraogo, B. Lankoandé, } \\
\text { A. Ouédraogo }\end{array}$ \\
\hline $\begin{array}{l}\text { Écriture - Préparation } \\
\text { de l'ébauche originale }\end{array}$ & B. Kabré \\
\hline $\begin{array}{l}\text { Écriture - Révision } \\
\text { et édition }\end{array}$ & $\begin{array}{l}\text { B. Kabré, M. Belem/Ouédraogo, } \\
\text { B. Lankoandé, A. Ouédraogo }\end{array}$ \\
\hline
\end{tabular}

Bois et Forêts des Tropiques - Revue scientifique du Cirad (c) Bois et Forêts des Tropiques (c) Cirad

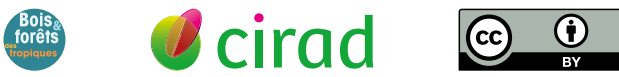

Cirad - Campus international de Baillarguet, 34398 Montpellier Cedex 5, France - Contact : bft@cirad.fr - ISSN : L-0006-579X 